\title{
Can the novel individualized-ultrasound method perform well in the subsequent follow-up of rheumatoid arthritis patients?
}

\author{
Wenxue $\mathrm{Li}^{1} \cdot$ Jiaan $\mathrm{Zhu}^{1}$ (D) \\ Received: 1 October 2019 / Accepted: 11 October 2019 / Published online: 22 October 2019 \\ (c) Japan Radiological Society 2019
}

Accurate assessment of joint inflammation and damage in rheumatoid arthritis (RA) is important for monitoring therapeutic effects and for predicting the outcome of the disease. To date, a number of ultrasound scoring methods have been performed $[1,2]$. However, at the patient level, there is absence of a standard ultrasound scoring method achieving general consensus. We are pleased to read the interesting article entitled "Detecting joints with erosion(s) in rheumatoid arthritis: a novel individualized-ultrasound method performs better than existing methods" by Dr. Tan et al. [3] in the Japanese Journal of Radiology. This study demonstrated that a novel individualized-ultrasound (IUS) method which selects up to 7 or 14 ultrasonographically could detect substantially more joints with erosions in RA patients versus existing methods on pre-fix 7 or 14 joints for ultrasonography. However, we would like to point out two concerns.

First, it is universally known that reduced joint ultrasound assessment including the most frequently involved joints can be easier and less time consuming to perform in daily management and clinical trials. In this context, Tan et al. proposed the novel IUS method compared to existing methods assessing fewer joints. Furthermore, Dr. Tan et al. also mentioned that the novel IUS method requires more time in the discussion. Is the statement of introduction inconsistent with that of discussion about the examination time? Could the authors offer the examination time of the two ultrasound methods?

Moreover, Tan et al. mentioned that they have presented data at only one time-point. So how do we apply this novel method to follow-up? The first method still needs rescanning the 36 joints and selecting 7 or 14 most affected joints ultrasonographically to achieve more accurate disease activity. However, this follow-up method is more time consuming.

Jiaan Zhu

zhujiaan@pkuph.edu.cn

1 Department of Ultrasound, Peking University People's Hospital, No. 11 Xizhimen South Street, Beijing 100044, China
The second method only needs rescanning the selected 7 or 14 joints at the baseline scan. It can be seen that Dr. Tan et al. used the second method based on this study and a previous study of Dr. Tan et al. [4]. Although subsequent follow-up re-scanning of the selected target joints will be less time consuming, the selected joints in the re-scanning may not reflect the true disease activity due to the different affected joints at the different time points of RA patients. Therefore, in order to assess whether the novel IUS perform well in the subsequent follow-up, future longitudinal studies comparing the novel and pre-fixed approaches, the two ways of the novel method at multiple time-points will be required.

\section{Compliance with ethical standards}

Conflict of interest The authors declare that they have no conflict of interest.

\section{References}

1. Hammer HB, Kvien TK. Comparisons of 7- to 78-joint ultrasonography scores: all different joint combinations show equal response to adalimumab treatment in patients with rheumatoid arthritis. Arthritis Res Ther. 2011;13:R78.

2. Hammer HB, Kvien TK, Terslev L. Ultrasound of the hand is sufficient to detect subclinical inflammation in rheumatoid arthritis remission: a post hoc longitudinal study. Arthritis Res Ther. 2017;19:221.

3. Tan YK, Li H, Allen JC, Thumboo J. Detecting joints with erosion(s) in rheumatoid arthritis: a novel individualized-ultrasound method performs better than existing methods. Jpn J Radiol. 2019;2019:1-5.

4. Tan YK, Allen JC Jr, Lye WK, Conaghan PG, D’Agostino MA, Chew LC, et al. Novel ultrasound joint selection methods using a reduced joint number demonstrate inflammatory improvement when compared to existing methods and disease activity score at 28 joints. J Rheumatol. 2016;43:34-7.

Publisher's Note Springer Nature remains neutral with regard to jurisdictional claims in published maps and institutional affiliations. 